\section{Adaptação para o Português da seção A do Cambridge Examination for Mental Disorders of the Elderly - Revised Version (CAMDEX-R) para o diagnóstico de demência}

\author{
Cross-cultural adaptation of section A of the \\ Cambridge Examination for Mental Disorders of \\ the Elderly - Revised Version (CAMDEX-R) for \\ dementia diagnosis
}

Daniele Aguiar Lima 1,2 Roberto Alves Lourenço 1

\footnotetext{
${ }^{1}$ Universidade do Estado do Rio de Janeiro, Rio de Janeiro, Brasil.

2 Hospital dos Servidores do Estado, Ministério da Saúde,

Rio de Janeiro, Brasil.

Correspondência

D. A. Lima

Universidade do Estado do Rio

de Janeiro.

Rua Fábio da Luz 214, apto.

702, Rio de Janeiro, $R J$

20720-350, Brasil.

dradanielelima@gmail.com
}

\section{Abstract}

This article concerns the first phase of the crosscultural equivalence of Section A in the Cambridge Examination for Mental Disorders of the Elderly Revised Version (CAMDEX-R), a clinical interview for dementia diagnosis. Evaluating conceptual and item equivalences involved a literature review and expert groups. Semantic equivalence in volved translations of the original into Portuguese and back-translations, with analysis of the versions and the original text; meetings with experts, discussions with 52 elders using the focus group technique; further meeting with experts; pretesting on 35 elders; and elaboration of the final version. For the reliability analysis, the version was applied to 160 patients. Inter-observer reliability was nearly perfect in all sub-sections of present state and in individual items of past medical history. Test-retest reliability was moderate or higher in the sub-sections and substantial or nearly perfect in $70 \%$ of the items in past history. The Brazilian version shows satisfactory equivalence to the original and good reliability levels.

Questionnaires; Translating; Reproducibility of Results; Dementia

\section{Introdução}

As demências se tornaram um importante problema de saúde pública devido ao aumento da sua prevalência, associado à transição demográfica e epidemiológica; aos seus aspectos clínicos, geradores de incapacidade cognitiva e funcional; e a alterações comportamentais ${ }^{1}$. A síndrome demencial produz seus efeitos também sobre os cuidadores dos pacientes, em decorrência da perda de produtividade e das consequências sobre a própria saúde daqueles 2 .

Estima-se que a prevalência das demências em indivíduos com 65 ou mais anos de idade, nos países desenvolvidos, seja de $6 \% 3$. No Brasil, além da escassez de dados epidemiológicos, a inexistência de informações que contemplem todo o território nacional e as dificuldades metodológicas nos estudos populacionais existentes dificultam conclusões abrangentes. No Estado de São Paulo, a prevalência média de quadros demenciais foi 7,1\% 4,5, sendo a doença de Alzheimer a causa mais comum.

Segundo os critérios do Diagnostic and Statistical Manual of Mental Disorders (DSM-IV), da Associação Americana de Psiquiatria 6, a característica essencial da demência é o desenvolvimento de múltiplos déficits cognitivos, que incluem comprometimento da memória e pelo menos uma das seguintes alterações cognitivas: afasia, apraxia, agnosia ou alteração do funcionamento executivo. Os déficits cognitivos comprometem 
o funcionamento ocupacional ou social e representam um declínio em relação ao nível prévio de funcionamento. O diagnóstico não deve ser feito se esses déficits ocorrem exclusivamente durante o curso de um delirium.

A síndrome demencial é muitas vezes subdiagnosticada 7 por causa das dificuldades no diagnóstico diferencial entre as alterações fisiológicas do envelhecimento, o déficit cognitivo leve e a fase inicial da doença ${ }^{8}$. Além disso, o quadro demencial pode, frequentemente, interferir na percepção subjetiva dos déficits pelo indivíduo ${ }^{9}$.

O diagnóstico de demência é essencialmente clínico e não há ainda um marcador biológico que seja capaz de defini-la claramente. A entrevista clínica é um dos principais instrumentos para o diagnóstico da doença 10 . Uma avaliação clínica, cognitiva, comportamental, funcional e social deve ser obtida quando houver queixa de perda de memória, de confusão mental ou de um declínio na habilidade para desempenhar tarefas cotidianas 11 . Os testes estruturados, sejam as entrevistas com o paciente e com o informante, sejam os testes neuropsicológicos, são bastante valiosos para uma completa investigação diagnóstica.

O Cambridge Examination for Mental Disorders of the Elderly - Revised Version (CAMDEX-R) é um instrumento estruturado para a abordagem clínica dos pacientes sob investigação de demência. É composto por nove seções e inclui a entrevista com o paciente e com o informante, a bateria de testes neuropsicológicos, exame físico e exames complementares. A adaptação transcultural do teste neuropsicológico e da entrevista com o informante contidos nesse exame foi realizada em projetos de outros autores 12,13, como parte de um programa de investigação para validação de instrumentos de avaliação cognitiva e funcional em idosos ambulatoriais. As demais seções do CAMDEX-R, referentes aos exames e informações adicionais da avaliação, pelas características próprias do instrumento, não sofrem interferência cultural e serão objeto de avaliação das equivalências conceitual, de itens, semântica e operacional, processo já em andamento.

O presente artigo tem como objetivo descrever o processo de adaptação transcultural da versão para o português da entrevista clínica com o indivíduo (seção A) do CAMDEX-R e determinar a confiabilidade interavaliador e teste-reteste, como parte deste processo.

Desta forma, após conclusão da equivalência de mensuração da seção A, ela irá compor, junto do trabalho realizado com as demais seções, a versão brasileira do CAMDEX-R, a ser oportunamente publicada.

\section{Metodologia}

\section{$O$ instrumento}

O Cambridge Examination for Mental Disorders of the Elderly (CAMDEX) foi desenvolvido e publicado em 1986, com a proposta de diagnosticar casos de demência em idosos, desenvolver medições confiáveis da extensão e gravidade do déficit cognitivo, além de avaliar as alterações comportamentais e a adaptação do indivíduo às atividades de vida diária 14 .

A versão revisada do instrumento, CAMDEX-R, foi estruturada pelo mesmo grupo de Geriatria e Psicogeriatria de Cambridge 15 e incorporou ao teste o diagnóstico diferencial de outros tipos de demência, tais como a demência dos corpos de Lewy e a demência frontal 16,17,18. Trata-se de instrumento extenso, já que é composto de 391 itens. Por esta razão, não se constitui um teste para rastreio, mas, sim, para a avaliação dos casos onde há dúvida diagnóstica.

A seção A, objeto do presente estudo, é a entrevista com o indivíduo. A seção B é a avaliação cognitiva e constitui o Cambridge Cognitive Examination na versão revisada (CAMCOG-R). É uma bateria neuropsicológica breve, composta por 72 itens. Os escores dos subitens podem ser interpretados separadamente, obtendo a noção de cada domínio cognitivo isolado, e o escore total demonstra a cognição de um modo global. No estudo de confiabilidade e validade da versão brasileira do CAMCOG-R, o melhor ponto de corte para a população total foi $60 / 61$, com $88 \%$ de sensibilidade e $84 \%$ de especificidade 12 . Na seção C, são relatadas as observações do entrevistador, e o exame físico é descrito na D. A seção E consiste nos registros dos exames laboratoriais e radiológicos, e a seção $\mathrm{F}$ registra todas as medicações em uso. A seção G corresponde às informações extras obtidas durante o atendimento. A seção H apresenta a entrevista com o informante, a qual compreende a investigação da alteração cognitiva, comportamental e funcional do paciente junto com um familiar ou algum acompanhante que o conheça bem ${ }^{13}$. Na seção I, é registrada qualquer informação relevante que não se enquadrou às perguntas estruturadas prévias.

A seção A corresponde a uma entrevista estruturada para a abordagem clínica dos pacientes. É composta por subseções com perguntas direcionadas a obter informações sobre o seu estado físico e mental atual, incluindo avaliação das funções cerebrovasculares, do sono, de distúrbio do humor e investigação das atividades funcionais cotidianas. A seguir, é pesquisada sua história patológica pregressa e a história familiar 
além de fatores de risco, manifestações psiquiátricas e tratamentos realizados.

Esta entrevista não foi constituída para ser uma escala quantitativa e não há ponto de corte para o diagnóstico. Ela é organizada para elaboração de uma impressão clínica. Compõe-se de 124 itens, numerados de 14 a 138, e deve ser aplicada face a face por um profissional com experiência no diagnóstico de demência.

\section{Primeira etapa: equivalências conceitual de itens, semântica e operacional}

Após autorização para uso do CAMDEX-R por parte do autor responsável pelo trabalho original, o projeto foi submetido e aprovado pela Comissão de Ética em Pesquisa do Hospital Universitário Pedro Ernesto, da Universidade do Estado do Rio de Janeiro (CEP/HUPE/UERJ).

Na primeira etapa do trabalho, dentro da abordagem universalista proposta por Herdman et al. 19, foram aplicadas estratégias no sentido de se buscarem as equivalências conceitual, de itens e semântica 20,21,22.

Durante a avaliação da equivalência conceitual e de itens, foi realizada uma extensa revisão bibliográfica, baseada na investigação dos fundamentos teóricos e do histórico de desen- volvimento e aplicação do instrumento. Foram realizadas discussões com especialistas da área de geriatria e gerontologia para avaliar se as dimensões abarcadas pelo exame original eram relevantes na cultura-alvo e se havia pertinência dos itens em captar cada uma dessas dimensões no contexto brasileiro.

$\mathrm{O}$ instrumento original foi submetido à tradução para o português em duas versões independentes, realizadas por dois brasileiros fluentes nos dois idiomas. As versões em português foram retrotraduzidas para o inglês por outros dois profissionais independentes (um inglês e um brasileiro), os quais não tiveram acesso ao instrumento original. A quinta avaliadora, brasileira e tradutora juramentada, elaborou a versãosíntese, com base na comparação entre a entrevista original, as traduções e retrotraduções, sem ter tido qualquer contato com os profissionais das etapas anteriores.

Um grupo multidisciplinar da área de geriatria e gerontologia, com amplo conhecimento dos critérios de demência, do CAMDEX-R e da língua inglesa, analisou a versão-síntese. O objetivo dessa etapa foi de melhor adequar as questões à população-alvo, mas sem alterar o significado geral de cada pergunta do instrumento.

Tabela 1

Itens abordados nos grupos focais.

\begin{tabular}{|c|c|c|}
\hline Subseção & Item & Objeto \\
\hline \multirow[t]{3}{*}{ Identificação } & 15 & Idade atual \\
\hline & 18 & Nível de escolaridade \\
\hline & 19 & Moradia \\
\hline Função cerebrovascular & 23 & Déficit neurológico focal \\
\hline Humor deprimido & 48 & Ideação ou tentativa suicida \\
\hline \multirow[t]{3}{*}{ Ansiedade } & 50 & Irritabilidade \\
\hline & 52 & Ansiedade \\
\hline & 54 & Ataque de pânico \\
\hline \multirow[t]{5}{*}{ Atividades diárias } & 56 & Dificuldade para iniciar movimento \\
\hline & 57 & Lentidão da marcha \\
\hline & 59 & Alteração da expressão facial \\
\hline & 61 & Dificuldade para realizar tarefas domésticas \\
\hline & 63 & Incontinência urinária \\
\hline \multirow[t]{4}{*}{ Paranoia e características psicóticas } & 74 & Alucinações auditivas \\
\hline & 80 & Delírio de perseguição \\
\hline & 82 & Delírio \\
\hline & 84 & Delírio hipocondríaco ou niilista \\
\hline \multirow[t]{2}{*}{ Histórico médico passado } & 97 & Uso de psicotrópicos \\
\hline & 101 & Inconsciência por reação adversa a medicamento \\
\hline Histórico médico familiar & 109 & Organização familiar (irmãos) \\
\hline
\end{tabular}


As questões que apresentaram maior dificuldade para adaptação, identificadas pelos especialistas na etapa anterior, foram selecionadas para discussão em grupos focais. No total, 20 itens foram discutidos nas reuniões (Tabela 1).

Os grupos focais foram constituídos de indivíduos acima de 60 anos, com boa capacidade cognitiva e funcional, selecionados dentre os pacientes de um ambulatório público de geriatria e dentre os participantes de um centro de convivência público para idosos. Os convites para participação nos grupos focais foram realizados por via telefônica, tendo $62 \%$ comparecido à reunião. Foram realizados sete grupos, totalizando 52 idosos; sua duração média foi de 77 minutos. A média de idade encontrada nos grupos foi de $72,8$ anos ( $\mathrm{DP}=5,02)$; a média de escolaridade foi de 6,9 anos (DP = 4,24).

As questões discutidas com os idosos nos grupos focais foram, posteriormente, analisadas pelo grupo multidisciplinar da geriatria, considerando, principalmente, a aceitabilidade de cada item e as sugestões elaboradas, resultando em uma nova versão.

Esta versão pré-teste foi aplicada em 35 idosos, oriundos do ambulatório e do centro de convivência. Foram excluídos os indivíduos que apresentavam quadro de demência, depressão ou déficit sensorial graves, ou qualquer outra doença incapacitante que prejudicasse a comunicação com a entrevistadora. Além de responderem a todas as perguntas do instrumento, os idosos foram questionados quanto à compreensão e pertinência dos itens. Foram, ainda, encorajados a parafrasear as perguntas consideradas mais difíceis e, em algumas situações, a justificar suas respostas. A duração média dos pré-testes foi de 35 minutos. A média de idade foi de 76,7 anos (DP $=6,72$ ), e a média de escolaridade encontrada, 5,9 anos (DP = 4,23).

As questões mais problemáticas foram discutidas novamente pelo grupo multidisciplinar de especialistas, que concluiu esta etapa do estudo e elaborou a versão final.

\section{Segunda etapa: confiabilidade}

O estudo foi realizado em um ambulatório público de geriatria, no período de março a agosto de 2008, com uma amostra de conveniência. Os pacientes eram convidados a participar no dia do atendimento médico ou de outra especialidade gerontológica. Os critérios de inclusão foram: ter 60 anos ou mais; ser brasileiro e ter o Mini-Exame do Estado Mental igual ou maior que 14 pontos. Foram excluídos os pacientes com quadro de $d e$ lirium, deficiência sensorial grave, diagnóstico de demência ou depressão graves, ou doença clínica grave ou descompensada.

Cento e sessenta indivíduos concordaram em participar, assinaram o termo de consentimento livre e esclarecido e foram submetidos à entrevista face a face, a qual também foi gravada em áudio. Um auxiliar de pesquisa (AP1) realizava a entrevista diretamente com o paciente e preenchia sua folha de respostas; outro (AP2) preenchia as respostas ouvindo as gravações, sem o conhecimento do formulário preenchido pelo AP1. Foi realizado rodízio entre os APs.

Em 130 indivíduos, as gravações foram consideradas adequadas, e esta foi a amostra com que se calculou a confiabilidade interavaliador do teste. Todos os idosos foram convidados a retornar para uma nova testagem com o mesmo auxiliar de pesquisa, em um período aproximado de 30 dias, para evitar o efeito de memória e a influência de mudanças no quadro clínico do indivíduo. Noventa e três deles completaram o estudo de confiabilidade teste-reteste. Em virtude de dificuldades de locomoção, alguns retornaram em um período de até 75 dias.

Os auxiliares de pesquisa foram duas assistentes sociais, um fisioterapeuta e uma médica geriatra, que foram submetidos a sessões de treinamento para padronização da entrevista. Um manual para aplicação da seção A foi produzido pelos pesquisadores responsáveis, com orientações sobre a aplicação, a codificação das respostas, o preenchimento da folha de respostas e a introdução das subseções e itens.

A confiabilidade foi calculada para cada subseção do instrumento. As subseções analisadas foram: distúrbios do sono (itens 24 a 26), humor deprimido (itens 27 a 48), ansiedade (itens 49 a 55), cognição (itens 64 a 70, referentes à memória; itens 71 a 73 , referentes ao funcionamento mental global) e paranoia e características psicóticas (itens 74 a 87).

Para a avaliação da presença da característica em cada subseção, consideraram-se os critérios do DSM-IV como padrão-ouro 6 .

A subseção função cerebrovascular (itens 20 a 23) inclui questões relativas a sintomas de acidente vascular encefálico, cefaleia, tonteiras e quedas; a subseção atividades diárias (itens 56 a 63) abrange questões de parkinsonismo e de atividades básicas e instrumentais da vida diária. Estas subseções não foram avaliadas no seu conjunto, pois as questões são muito distintas entre si e não é possível concluir um diagnóstico ao serem analisadas separadamente. As questões relativas ao histórico médico passado (parte 2), também por sua diversidade, já que investigam doenças e fatores de risco distintos, foram avaliadas isoladamente item a item. Os demais itens 
referentes à identificação (itens 14 a 19) e histórico médico familiar (parte 3) não foram submetidos aos testes de confiabilidade.

Na análise dos dados, foram utilizados o índice kappa (k) e os critérios de Landis \& Koch 23 para a sua interpretação: quase perfeita, $>0,80$; substancial, de 0,61 a 0,80; moderada, de 0,41 a 0,60 ; regular, de 0,21 a 0,40 ; fraca, de 0,01 a 0,20 ; pobre, 0 ou menos. Os dados foram armazenados e analisados por meio do programa SPSS versão 12.0 (SPSS Inc., Chicago, Estados Unidos).

\section{Resultados}

Após a primeira etapa do estudo, referente às equivalências conceitual, de itens, semântica e operacional, 92 dos 124 itens do instrumento original não sofreram outras modificações, além daquelas produzidas durante o processo de tradução, retrotradução e elaboração da versão-síntese. As maiores alterações foram realizadas nos itens 15, 19 e 109. Estes itens englobam dados sobre idade, moradia e ordem cronológica dos irmãos, respectivamente, porém as estruturas gerais das perguntas no instrumento original não se aplicavam à população-alvo.

A pergunta 19, por exemplo, foi uma das que mais gerou discordância, uma vez que utiliza termos que não são usados de forma distinta na cultura brasileira, que são: nursing home, residential home e sheltered accommodation. Na prática, todos são usados como instituições asilares. $\mathrm{Na}$ discussão com os idosos pela técnica de grupos focais, "residencial de idosos" foi avaliado como um tipo de acomodação onde os residentes são independentes e com boas condições socioeconômicas, enquanto "lar de idosos" foi considerado como referente a instituições precárias, com acomodação de residentes com distúrbio cognitivo e/ou funcional. Nas discussões posteriores com os especialistas, optou-se por utilizar os critérios do estudo sobre instituições de longa permanência para idosos (ILPI), da pesquisadora Ana Amélia Camarano 24. Consideraram-se apenas duas categorias: asilo, englobando instituições públicas, privadas filantrópicas e mistas, e residencial de idosos, para instituições privadas com fins lucrativos.

Vinte e quatro itens foram parcialmente modificados durante o processo de equivalência semântica (Tabela 2). A expressão "heavy smoker" da pergunta 93, por exemplo, foi retirada devido ao estigma de "fumante pesado ou inveterado", tendo sido substituída por "O senhor já fumou?". Do mesmo modo, a expressão "heavy drinker", encontrada na pergunta 95, gerou dúvida pelo fato de ser difícil traduzi-la para uma expressão equivalente e que não causasse constrangimento (como, por exemplo, alcoólatra). Optou-se, então, pela reformulação da pergunta para “ $O \operatorname{Sr}(a)$ já achou que bebia demais?".

Cinco itens das subseções de identificação, função cerebrovascular, sono e humor deprimido foram desmembrados para melhor compreensão dos indivíduos entrevistados (itens 18, 23, 26, 27 e 28).

Durante os testes de confiabilidade, a duração das entrevistas variou de 15 a 55 minutos, com média de 30 minutos, tanto na primeira aplicação do instrumento, quanto no reteste.

Não foram observadas diferenças significativas no perfil sociodemográfico dos indivíduos analisados para a confiabilidade interaferidor e daqueles que retornaram para o reteste, mediante a realização do teste qui-quadrado $(\mathrm{p}<0,05)$.

Os participantes do estudo se constituíram predominantemente de mulheres (acima de $70 \%$ dos indivíduos), com idade avançada (65\% acima de 70 anos) e com mais de $60 \%$ da amostra com escolaridade igual ou inferior a quatro anos e renda mensal até um salário-mínimo (Tabela 3).

A confiabilidade interavaliador foi quase perfeita em todos os domínios das subseções avaliadas. Em relação ao teste-reteste, a concordância foi substancial nas subseções humor deprimido, cognição e paranoia e características psicóticas. Nas subseções distúrbio do sono e ansiedade, o kappa encontrado foi moderado (Tabela 4).

Em relação aos itens individuais do histórico médico passado, a confiabilidade interavaliador foi quase perfeita em todas as questões, e o índice kappa variou de 0,839 a 1,00. A confiabilidade teste-reteste foi quase perfeita em três itens, substancial em quatro deles e moderada em dois.

\section{Tabela 2}

Itens parcialmente modificados no processo de equivalência semântica.

\begin{tabular}{lc}
\hline Subseção & Itens \\
\hline Função cerebrovascular & 20,21 \\
Sono & $24,25,26$ \\
Humor deprimido & $29,32,37,48$ \\
Ansiedade & 52 \\
Atividades de vida diária & 61,63 \\
Memória & $65,67,69$ \\
Paranoia e características psicóticas & $74,76,82,84$ \\
Histórico médico passado & $88,90,93,94,95$
\end{tabular}


Tabela 3

Características sociodemográficas dos participantes do estudo de confiabilidade.

\begin{tabular}{|c|c|c|c|c|}
\hline & \multicolumn{2}{|c|}{ Interavaliador $(n=130)$} & \multicolumn{2}{|c|}{ Teste-reteste $(n=93)$} \\
\hline & $\mathrm{n}$ & $\%$ & $\mathrm{n}$ & $\%$ \\
\hline \multicolumn{5}{|l|}{ Gênero } \\
\hline Masculino & 36 & 27,7 & 25 & 26,9 \\
\hline Feminino & 94 & 72,3 & 68 & 73,1 \\
\hline \multicolumn{5}{|l|}{ Idade (anos) } \\
\hline $60-64$ & 4 & 3,1 & 3 & 3,2 \\
\hline $65-69$ & 15 & 11,5 & 12 & 12,9 \\
\hline $70-74$ & 26 & 20,0 & 18 & 19,4 \\
\hline $75-79$ & 35 & 26,9 & 32 & 34,4 \\
\hline $80-84$ & 30 & 23,1 & 20 & 21,5 \\
\hline$>85$ & 20 & 15,4 & 8 & 8,6 \\
\hline \multicolumn{5}{|l|}{ Estado civil } \\
\hline Solteiro & 16 & 12,3 & 15 & 16,1 \\
\hline Casado & 43 & 33,1 & 33 & 35,5 \\
\hline Separado & 8 & 6,2 & 8 & 8,6 \\
\hline Viúvo & 63 & 48,5 & 37 & 39,8 \\
\hline \multicolumn{5}{|c|}{ Escolaridade (anos) } \\
\hline Analfabeto & 15 & 11,5 & 8 & 8,6 \\
\hline $1-4$ & 68 & 52,3 & 50 & 53,8 \\
\hline $5-8$ & 29 & 22,3 & 19 & 20,4 \\
\hline $9-11$ & 13 & 10,0 & 12 & 12,9 \\
\hline$>12$ & 5 & 3,8 & 4 & 4,3 \\
\hline \multicolumn{5}{|c|}{ Renda (salários mínimos) } \\
\hline Até 1 & 78 & 60,0 & 56 & 60,2 \\
\hline $2-4$ & 47 & 36,2 & 35 & 37,6 \\
\hline$>5$ & 5 & 3,8 & 2 & 2,2 \\
\hline
\end{tabular}

Tabela 4

Confiabilidade interavaliador e teste-reteste das subseções da seção A do Cambridge Examination for Mental Disorders of the Elderly - Revised Version (CAMDEX-R).

\begin{tabular}{llc}
\hline Subseção/Domínio & Confiabilidade interavaliador (kappa) & Confiabilidade teste-reteste (kappa) \\
\hline Distúrbio do sono & 0,984 & 0,477 \\
Humor deprimido & 0,951 & 0,629 \\
Ansiedade & 0,908 & 0,579 \\
Cognição & 0,938 & 0,697 \\
Paranoia & 0,948 & 0,637 \\
\hline
\end{tabular}

Apenas no item 100, relativo a efeito colateral grave (síndrome neuroléptica maligna) com uso de um novo medicamento, o kappa foi regular (Tabela 5).

\section{Discussão}

Este estudo faz parte de um programa de investigação de instrumentos para avaliação cognitiva e funcional em idosos. O processo de adaptação 
Confiabilidade interavaliador e teste-reteste dos itens do histórico médico passado da seção A do Cambridge Examination for Mental Disorders of the Elderly - Revised Version (CAMDEX-R).

\begin{tabular}{lccc}
\hline Item & Objeto & $\begin{array}{c}\text { Confiabilidade } \\
\text { interavaliador (kappa) }\end{array}$ & $\begin{array}{c}\text { Confiabilidade teste-reteste } \\
\text { (kappa) }\end{array}$ \\
\hline 88 & Infarto agudo do miocárdio & 0,956 & 0,754 \\
89 & Hipertensão arterial & 0,914 & 0,768 \\
90 & Acidente vascular encefálico & 1,00 & 0,591 \\
91 & Síncope por traumatismo crânio-encefálico & 0,967 & 0,691 \\
92 & Convulsões ou epilepsia & 0,905 & 0,852 \\
93 & Tabagismo & 0,839 & 0,877 \\
94 & Etilismo & 0,905 & 0,555 \\
97 & Uso de psicotrópicos & 1,00 & 0,726 \\
99 & Doença psiquiátrica & 0,974 & 0,825 \\
100 & Reação adversa a medicamento & 1,00 & 0,354 \\
\hline
\end{tabular}

transcultural para o Brasil do CAMDEX-R está em andamento, e as nove partes que o constituem receberam atenção diferenciada, segundo o julgamento dos autores, em virtude do maior ou menor grau de interferência que o instrumento sofreria no processo de adaptação. Um procedimento semelhante de adaptação transcultural descrito no presente artigo, através do modelo de equivalências, também foi aplicado às seções $\mathrm{B}$ (avaliação cognitiva) e $\mathrm{H}$ (entrevista com o informante) 12,13 .

Por ser a primeira tradução sistemática da seção A do CAMDEX-R para o português falado no Brasil, após as etapas de tradução, retrotradução, discussão com especialistas, técnica de grupos focais e pré-teste, foram realizadas modificações em 32 itens, a fim de adequar o instrumento para a realidade do idoso brasileiro. Posteriormente, a versão final foi submetida aos testes de confiabilidade, tendo sido encontrados resultados satisfatórios na confiabilidade interavaliador e teste-reteste, o que reflete a estabilidade desta versão. Somente em um item, relativo à síndrome neuroléptica maligna (item 100), a confiabilidade teste-reteste foi regular, provavelmente por abordar uma patologia muito específica e rara, com uma pergunta considerada vaga pelos pesquisadores. Portanto, o principal aspecto positivo do presente trabalho é o fato de que esta versão da seção A foi obtida por meio de um cuidadoso processo de adaptação transcultural, compatível com as rigorosas recomendações metodológicas atuais 25 .

O CAMDEX-R é um instrumento extenso, cujo objetivo principal é auxiliar no diagnóstico de demência, estando sua aplicação restrita a ca- sos selecionados por suspeita clínica, e não deve ser usado como instrumento de rastreio. A versão revisada apresenta melhor sensibilidade e especificidade em relação à sua versão original 17. Por essas características, cada vez mais se fortalece a proposta de utilizá-lo como instrumento de definição diagnóstica, tanto em ambiente clínico, quanto em pesquisa epidemiológica.

Os altos valores obtidos na confiabilidade interavaliador sugerem que o instrumento pode ser aplicado por profissionais de saúde não médicos, desde que tenham conhecimento dos critérios de demência e do seu diagnóstico diferencial. Além disso, devem receber treinamento adequado para aplicação da entrevista.

Da mesma forma, a entrevista clínica do CAMDEX-R também pode ser útil no treinamento em geriatria como guia para a anamnese dos pacientes sob suspeita de déficit cognitivo. Essa possibilidade de utilização do instrumento torna-o um forte candidato a ser incorporado às estratégias de ensino acadêmico das várias profissões e especialidades que lidam com transtornos cognitivos.

Um aspecto metodológico merece consideração quando se examina a confiabilidade de um processo de aferição. Os entrevistadores, cientes do processo de avaliação em curso, tendem a proceder com maior rigor do que em circunstâncias habituais, o que pode superestimar os resultados da confiabilidade interavaliador. Naturalmente, este é um aspecto inerente a qualquer procedimento científico: o isolamento dos fenômenos nos laboratórios de pesquisa acaba por criar situações ideais de estudo, muitas vezes produzindo resultados diferentes daqueles obtidos em situa- 
ções ecológicas. No entanto, os autores não acreditam que isso se constitua num ponto negativo, já que esses resultados devem ser vistos, no que tange à confiabilidade do instrumento, como a tradução do potencial de reprodutibilidade inerente a ele.

Durante todo o processo de equivalência conceitual, de itens e semântica, um problema amplamente discutido foi a influência do nível sociocultural e da escolaridade na compreensão das questões pelos entrevistados. Dessa forma, várias adaptações foram realizadas para adequar os itens para uma população de baixa escolaridade. Sem dúvida, por essa razão, a versão final obtida deve ser vista como uma, entre muitas outras possíveis, considerando-se as peculiaridades da população de idosos que participaram do estudo. Portanto, consideramos que esta seja outra limitação do estudo: a maioria dos indivíduos tinha idade avançada, baixa escolaridade e frequentava um ambulatório de saúde da rede pública, tornando a amostra homogênea e não representativa do conjunto da população idosa brasileira. Sugere-se a aplicação desta versão em outros ambientes operacionais (como idosos que vivem na comunidade, atendidos em ambulatórios gerais, hospitalizados e internados em instituições asilares, por exemplo) e em populações com características demográficas diferentes (idosos mais jovens, com maior escolaridade e melhor nível socioeconômico, por exemplo), para melhor avaliação da sua aplicabilidade e estabilidade nessas outras circunstâncias.

Uma vez que a seção A é uma entrevista com o paciente que fornece uma impressão clínica em relação ao déficit cognitivo e sua aplicação está localizada no contexto do CAMDEX-R, seu uso isolado para diagnóstico de síndrome demencial ainda não pode ser recomendado. Para isto, novos estudos seriam necessários.

Embora o diagnóstico das subseções da história médica atual tenha sido baseado nos critérios do DSM-IV (depressão e distúrbio de ansiedade, por exemplo), não se pode recomendar apenas a utilização das subseções para o diagnóstico dessas doenças.

A confiabilidade das subseções foi moderada ou superior nos retestes, mas foi inferior à observada para interavaliador em todos os casos. Um dos fatores que podem ser responsáveis por isso foi o intervalo entre as entrevistas, o qual foi definido em 30 dias. Porém, em alguns casos, os pa- cientes retornaram posteriormente (até 75 dias depois), em virtude de dificuldade de locomoção, por fatores quer físicos, quer sociais, sendo esta outra limitação do estudo.

Há de se ressaltar que o intervalo de tempo entre os testes pode influenciar a confiabilidade teste-reteste. Por exemplo, se o teste é repetido em um curto intervalo de tempo, a memória da primeira aplicação do instrumento pode influenciar os resultados da segunda e, assim, falsamente inflar a confiabilidade teste-reteste. Opostamente, as mudanças no estado de saúde que ocorrem entre os testes podem influenciar as respostas dos entrevistados e diminuir a confiabilidade teste-reteste 26 . A confiabilidade obtida em vários estudos se mostrou progressivamente menor com o aumento do intervalo de reteste 27,28 . Como a história natural das demências é de anos de evolução e a confiabilidade teste-reteste foi satisfatória, os autores não excluíram os indivíduos que retornaram em um período maior que trinta dias. A dificuldade operacional de retorno deles também fez com que a amostra estudada para o reteste fosse menor do que para a estabilidade interavaliador. Apesar deste aspecto negativo, não há evidência de que esse fato tenha comprometido a qualidade dos resultados.

Uma desvantagem da seção A é sua extensão; muitos participantes (tanto do pré-teste, quanto do estudo de confiabilidade) queixaram-se do tempo de aplicação, o que também pode ter propiciado o não comparecimento ao reteste. Os pesquisadores observaram redundância em muitos itens, principalmente na terceira parte, relativa à história médica familiar. Um estudo posterior deverá avaliar uma versão resumida desta seção.

Outro estudo posterior a ser conduzido será o da equivalência de mensuração, englobando a validade de constructo e de critério deste instrumento. Essa etapa é fundamental para completar a equivalência funcional da versão brasileira do teste, a fim de que o instrumento possa ser utilizado fora do ambiente de pesquisa.

Concluindo, a versão final da seção A do CAMDEX-R apresentou níveis satisfatórios de estabilidade no tempo e entre os examinadores. Estudos adicionais, já em andamento, completarão esta pesquisa para que essa entrevista clínica possa ser utilizada no contexto linguístico-sóciocultural da população idosa brasileira. 


\section{Resumo}

Este artigo enfoca a primeira fase da adaptação transcultural da seção A do Cambridge Examination for Mental Disorders of the Elderly - Revised Version (CAMDEX-R), que é uma entrevista para o diagnóstico de demência. A avaliação da equivalência conceitual e de itens envolveu revisão bibliográfica e discussões com especialistas. A avaliação da equivalência semântica constou de: traduções do inglês para o português e retrotraduções, com análise das versões e do texto original; encontros com experts; discussão com 52 idosos através da técnica de grupos focais; nova reunião de especialistas; pré-teste com 35 idosos; produção da versão final. Para avaliar a estabilidade, a versão final foi aplicada a 160 pacientes. A confiabilidade interavaliador foi quase perfeita nas subseções do estado clínico atual e nos itens individuais da história patológica pregressa. A confiabilidade teste-reteste foi moderada ou superior nas subseções e foi quase perfeita ou substancial em $70 \%$ dos itens da história patológica progressa. A versão brasileira apresentou boa equivalência com o original e bons níveis de confiabilidade.

Questionários; Tradução (Processo); Reprodutibilidade dos Resultados; Demência

\section{Referências}

1. Morris J. Dementia update 2005. Alzheimer Dis Assoc Disord 2005; 19:100-17.

2. Garrido R, Menezes PR. Impacto em cuidadores de idosos com demência atendidos em um serviço psicogeriátrico. Rev Saúde Pública 2004; 38:835-41.

3. Rocca WA, Cha RH, Waring SC, Kokmen E. Incidence of dementia and Alzheimer's disease: a reanalysis of data from Rochester, Minnesota, 19751984. Am J Epidemiol 1998; 148:51-62.

4. Montaño MBM, Koening AM, Ramos LR. Prevalência de demência em uma coorte de idosos da comunidade. In: Anais da III Reunião de Pesquisadores em Doenças de Alzheimer e Desordens Relacionadas. São Paulo: Academia Brasileira de Neurologia; 2001. p. 2-3.

5. Herrera Jr. E, Caramelli P, Nitrini R. Estudo epidemiológico populacional de demência na cidade de Catanduva, estado de São Paulo, Brasil. Rev Psiquiatr Clín (São Paulo) 1998; 25:70-3.

\section{Colaboradores}

Ambos os autores participaram do processo de planejamento da investigação, coleta e análise de dados, e redação do artigo.
6. American Psychiatric Association. Diagnostic and statistical manual of mental disorders - fourth edition. Washington DC: American Psychiatric Association; 1994.

7. Pisiani MA, Redlich C, McNicoll L, Ely EW, Inouye SK. Underrecognition of preexisting cognitive impairment by physicians in older ICU patients. Chest 2003; 124:2267-74.

8. Kukull WA, Bowen JD. Dementia epidemiology. Med Clin North Am 2002; 86:573-90.

9. Neri M, Roth M, De Vreese LP, Rubichi S, Finelli C, Bolzani R, et al. The validity of informant reports in assessing the severity of dementia: evidence from the CAMDEX interview. Dement Geriatr Cogn Disord 1998; 9:56-62.

10. Demers L, Oremus M, Perrault A, Wolfson C. Review of outcome measurement instruments in Alzheimer's disease drug trials: introduction. J Geriatr Psychiatry Neurol 2000; 13:161-9. 
11. Resnikoff D. Consenso sobre a síndrome de de terioração intelectual e demência: a experiência mexicana. In: Forlenza OV, Caramelli P, organizadores. Neuropsiquiatria geriátrica. São Paulo: Editora Atheneu; 2001. p. 53-64.

12. Paradela EMP. Adaptação transcultural para o português do Teste Cognitivo de Cambridge (CAMCOG-R) e desempenho do teste em idosos ambulatoriais [Tese de Doutorado]. Rio de Janeiro: Universidade do Estado do Rio de Janeiro; 2007.

13. Sampaio SGSM. Tradução, adaptação transcultural e confiabilidade de uma entrevista estruturada com informante para o diagnóstico de demência [Dissertação de Mestrado]. Rio de Janeiro: Universidade do Estado do Rio de Janeiro; 2007.

14. Roth M, Tym E, Mountjoy CO, Huppert FA, Hendrie $\mathrm{H}$, Verma S, et al. CAMDEX: a standardized instrument for the diagnosis of mental disorders in the elderly with special reference to the early detection of dementia. Br J Psychiatry 1986; 149:698-709.

15. Roth M, Huppert F, Mountjou CQ, Tym E. CAMDEX-R: The Cambridge Examination for Mental Disorders of the Elderly. Cambridge: Cambridge University Press; 1998.

16. Ballard CG, Ayre G, O'Brien J, Sahgal A, McKeith IG, Ince PG, et al. Simple standardised neuropsychological assessments aid in the differential diagnosis of dementia with Lewy bodies from $\mathrm{Al}$ zheimer's disease and vascular dementia. Dement Geriatr Cogn Disord 1999; 10:104-8.

17. Lozano-Gallego $\mathrm{M}$, Llinàs-Regà J, López-Pousa $\mathrm{S}$, Vilalta-Franch J. El CAMDEX-R en la evaluación clínica de las demencias. Actas Esp Psiquiatr 2000; 28:125-9.

18. Heinik J, Solomesh I. Validity of the Cambridge Cognitive Examination-Revised new executive function scores in the diagnosis of dementia: some early findings. J Geriatr Psychiatry Neurol 2007; 20:22-8.

19. Herdman M, Fox-Rushby J, Badia X. A model of equivalence in the cultural adaptation of HRQoL instruments: the universalist approach. Qual Life Res 1998; 7:323-35.
20. Moraes CL, Hasselmann MH, Reichenheim ME. Adaptação transcultural para o português do instrumento "Revised Conflict Tactics Scales (CTS2)" utilizado para identificar violência entre casais. Cad Saúde Pública 2002; 18:163-76.

21. Hasselmann MH, Reinchenheim ME. Adaptação transcultural da versão em português da Conflict Tactics Scales Form R (CTS-1), usada para aferir violência no casal: equivalências semântica e de mensuração. Cad Saúde Pública 2003; 19:1083-93.

22. Reichenheim ME, Moraes CL. Adaptação transcultural do instrumento Parent-Child Conflict Tactics Scales (CTSPC) utilizado para identificar a violência contra a criança. Cad Saúde Pública 2003; 19:1701-12.

23. Landis JR, Koch GG. The measurement of observer agreement for categorical data. Biometrics 1977; 33:159-74

24. Camarano AA, Andrade AO, Fernandes DF, Mello JLE, Christophe M, Epifano S, organizadores. Características das Instituições de Longa Permanência para Idosos - Região Sul. v. 3. Brasília: Instituto de Pesquisa Econômica Aplicada; 2008.

25. Reichenheim ME, Moraes CL. Operacionalização de adaptação transcultural de instrumentos de aferição usados em epidemiologia. Rev Saúde Pública 2007; 41:665-73.

26. Renosto A, Biz P, Hennington EA, Pattussi MP. Confiabilidade teste-reteste do Índice de Capacidade para o Trabalho em trabalhadores metalúrgicos do Sul do Brasil. Rev Bras Epidemiol 2009; 12:217-25.

27. McDowell I, Newell C. Measuring health. A guide to rating scales and questionnaires. $2^{\text {nd }}$ Ed. New York: Oxford University Press; 1996.

28. Lourenço RA, Veras RP, Ribeiro PCC. Confiabilidade teste-reteste do Mini-Exame do Estado Mental em uma população idosa assistida em uma unidade ambulatorial de saúde. Rev Bras Geriatr Gerontol 2008; 11:7-16.

Recebido em 21/Set/2009

Versão final reapresentada em 21/Jan/2010

Aprovado em 05/Abr/2010 